УДК 517.984.5

\title{
Решение спектральной задачи Рэлея резольвентным методом
}

\section{Е.М. Малеко ${ }^{1}$}

Аннотация: В работе рассматривается решение спектральной задачи Рэлея в случае, когда профиль скорости $q(x)$ - многочлен ненулевой степени. В этом случае спектр задачи может не содержать собственных чисел. Какой же он на самом деле рассмотрено в данной работе.

Ключевые слова: Спектр линейного оператора, собственные числа, дискретная часть спектра, остаточная часть спектра, задача Рэлея.

\section{1. ПОСТАНОВКА ЗАДАЧИ}

Задача Орра-Зоммерфельда в случае невязкой жидкости (число Рейнольдса $R \rightarrow \infty)$ вырождается в задачу, называемую задачей Рэлея:

$$
\left(q(x)-\frac{\lambda}{\alpha}\right)\left(D^{2}-\alpha^{2}\right) y=q^{\prime \prime}(x) y, \quad y=y(x),
$$

с условием

$$
y( \pm 1)=0 \text {. }
$$

Пусть $q(x)$ - многочлен ненулевой степени и такой, что

$$
\left\|\frac{q^{\prime \prime}(x)}{q(x)} \cdot M_{0}^{-1}\right\|<\infty
$$

Операторы $M_{0}, L_{1}, L_{2}$ представлены следующим образом:

$$
M_{0}:=D^{2}-\alpha^{2}, \quad L_{1}:=q^{\prime \prime}(x) \mathbb{I}, \quad L_{2}:=q(x) \mathbb{I}
$$

на областях определения

$$
\mathcal{D}\left(M_{0}\right)=\mathcal{D}\left(L_{1}\right)=\left\{y \mid y \in W_{2}^{2}[-1,1], y( \pm 1)=0\right\}, \quad \mathcal{D}\left(L_{2}\right)=\mathbb{H},
$$

где $W_{2}^{k}[-1,1], k \geq 0,-$ пространства Соболева.

\footnotetext{
${ }^{1}$ emaleko@rambler.ru
} 
Замена $M_{0} y=\phi$ сводит задачу (1)-(2) к виду

$$
q^{\prime \prime}(x) M_{0}^{-1} \phi-q(x) \phi=c \phi,
$$

где $\phi=\phi(x) \in \mathbb{H}=L_{2}(-1,1), c=-\lambda / \alpha \in \mathbb{C}$.

Оператор $M_{0}^{-1}$ является ядерным и его собственные числа равны $\mu_{n}^{-1 / 2} \sim n^{-2}$ при $n \rightarrow \infty$. Операторы $L_{1} M_{0}^{-1}$ и $L_{2}$ ограничены в $\mathbb{H}$, поэтому и $T:=\left(L_{1} M_{0}^{-1}-L_{2}\right)$ - также ограниченный в $\mathbb{H}$ оператор.

Таким образом, спектральная задача Рэлея заключается в вычислении сначала спектра $\sigma(T)$ оператора $T$ :

$$
T \phi=c \phi, \quad \phi \in \mathbb{H},
$$

а затем, с помощью замены $\lambda=-c \alpha$, в нахождении и спектра $€$ задачи (1)-(2).

\section{2. РЕШЕНИЕ ЗАДАЧИ}

Итак, пусть $\sigma(T)-$ спектр оператора $T, \sigma(T) \subset \mathbb{C}$. Из уравнения (4) легко получить следующее операторное уравнение

$$
\frac{q^{\prime \prime}(x)}{q(x)+c} M_{0}^{-1}=\mathbb{I}
$$

Дифференциальное выражение

$$
q(x)\left(D^{2}-\alpha^{2}\right) y(x)-q^{\prime \prime}(x) y(x)
$$

является самосопряжённым по Наймарку (см. [1, с. 14]), поэтому Tсамосопряжённый ограниченный оператор в $\mathbb{H}$ и его спектр лежит гдето на отрезке $[-\|T\|,\|T\|]$. Уточним его. Для этого запишем достаточно очевидное свойство А уравнения (6):

Из условия (3) и представления (6) следует, что для любого какого угодно малого $\delta_{0}$ и такого, что $2^{-p-2}>\delta_{0}>0$, любого комплексного $c$ множество

$$
U_{c, \delta_{0}}:=
$$




$$
\begin{gathered}
\{\phi(x) \in \mathbb{H}: \quad \phi(x)=0 \quad \text { n.в. на } \\
\mathfrak{M}:=\left(\bigcup_{i=1}^{p}\left[x_{i}-\delta_{0}, x_{i}+\delta_{0}\right]\right) \cap[-1,1], \\
\left.\mu(\mathfrak{M})<2, \quad\left\{x_{i}\right\}_{i=1, \ldots, p}=\mathfrak{q}^{-1}(-c) \cap[-1,1]\right\}
\end{gathered}
$$

включено в область определения оператора $q^{\prime \prime}(x) /(q(x)+c) \cdot M_{0}^{-1}$. Здесь $\mathfrak{q}^{-1}(-c)$ - прообраз точки $(-c)$ полинома $q(x) ; \mu(\mathfrak{M})=\sum_{k=1}^{s}\left|\bar{l}_{k}\right|-$ сумма длин непересекающихся подотрезков $\bar{l}_{k}$, составляющих $\mathfrak{M}: \mathfrak{M}=$ $\bigcup_{k=1}^{s} \bar{l}_{k}, \bar{l}_{i} \cap \bar{l}_{j}=\varnothing, i \neq j$. Если $\mathfrak{q}^{-1}(-c) \cap[-1,1]=\varnothing$, mо $U_{c, \delta_{0}}=\mathbb{H}$.

Используя стандартную процедуру построения, выпишем функцию Грина $G(x, \xi)\left(G(x, \xi)\right.$ - ядро оператора $\left.M_{0}^{-1}\right)$ :

$$
\begin{gathered}
G(x, \xi)= \\
\frac{1}{2 \alpha} \frac{(\exp (-\alpha(\xi-1))-\exp (\alpha(\xi-1)))(\exp (\alpha(x+1))-\exp (-\alpha(x+1)))}{\exp (-2 \alpha)-\exp (2 \alpha)}, \\
\text { для }-1 \leq x \leq \xi \leq 1 \text { и } G(x, \xi)= \\
\frac{1}{2 \alpha} \frac{(\exp (-\alpha(\xi+1))-\exp (\alpha(\xi+1)))(\exp (\alpha(-1+x))-\exp (-\alpha(-1+x)))}{\exp (-2 \alpha)-\exp (2 \alpha)}, \\
\text { для }-1 \leq \xi \leq x \leq 1 . \text { Ясно, что } \\
\left(M_{0}^{-1} \phi\right)(x)=\int_{-1}^{1} G(x, \xi) \phi(\xi) d \xi \quad \forall \phi \in \mathbb{H} .
\end{gathered}
$$

Уравнение (4) перепишем в виде

$$
q^{\prime \prime}(x) M_{0}^{-1} \phi(x)=(q(x)+c) \phi(x),
$$

где функция $\mathfrak{f}(x):=q^{\prime \prime}(x) M_{0}^{-1} \phi(x)$ на концах отрезка $[-1,1]$ равна нулю. А учитывая, что в качестве ортонормированного базиса в $\mathbb{H}$ можно принять набор

$$
\left\{y_{k}(x)=\frac{P_{k}(x)}{\left\|P_{k}(x)\right\|}\right\}, \quad k=0,1,2,3, \ldots,
$$


где $P_{k}(x)$ - многочлен Лежандра, любую функцию из $\mathbb{H}$ можно представить конечной или бесконечной линейной комбинацией из функций $y_{k}(x)$ (полиномов). Таким образом, если $\phi(x)$ - полином, то справа в (7) будет стоять полином, а слева, из вида функции Грина $G(x, \xi),-$ сумма полинома и полиномов, умноженных на экспоненты. То есть, если $q(x)=Q_{n}(x), \phi(x)=T_{m}(x)$ - полиномы $n$-й и $m$-й степеней соответственно, то (7) будет иметь следующий вид:

$$
\begin{aligned}
& Q_{n}^{(2)}(x)\left(T_{1, m}(x) e^{-2 \alpha x}+T_{2, m}(x) e^{-\alpha x}+T_{3, m}(x)+\right. \\
& \left.T_{4, m}(x) e^{\alpha x}+T_{5, m}(x) e^{2 \alpha x}\right)=\left(Q_{n}(x)+c\right) T_{m}(x),
\end{aligned}
$$

где $T_{i, m}(x)(i=1, \ldots, 5)$ - ненулевые полиномы степеней, не превосходящих $m$, так как они возникают в результате интегрирования по частям произведения многочлена $T_{m}(x)$ на функцию Грина. Если решение уравнения (8) искать в виде многочлена $\phi(x)=T_{m}(x)$, то $\phi(x) \equiv 0$. Другого полиномиального решения быть не может, так как в $(8)$ все $T_{i, m}(x)$ должны обнулиться, иначе, в случае ненулевой степени $m$ многочлена $T_{m}(x)$, будет отсутствовать равенство для п.в. $x \in[-1,1]$. Из всюду плотности множества всех многочленов в $\mathbb{H}$ можно сделать вывод, что другого (неполиномиального и ненулевого) решения в $\mathbb{H}$ уравнение (4) для любого $c \in[-\|T\|,\|T\|]$ иметь не может. В результате, для произвольно взятых $c \in[-\|T\|,\|T\|]$ резольвента $R_{c}(T)$ существует. А если учесть, что все точки из $\mathbb{C} \backslash[-\|T\|,\|T\|]$ являются регулярными для оператора $T$, то резольвента $R_{c}(T)$ существует для любого $c \in \mathbb{C}$.

Этот же факт можно доказать и по-другому, а именно: Уравнение (4) запишем в виде

$$
q^{\prime \prime}(x) M_{0}^{-1} \phi-(q(x)+\nu) \phi=(c-\nu) \phi,
$$

где $\phi=\phi(x) \in \mathbb{H}, c=-\lambda / \alpha, \nu>>1$. Тогда можно ввести в рассмотрение операторное уравнение

$$
R_{\nu}(T)=\tilde{c} \mathbb{I}, \quad \tilde{c}=\frac{1}{c-\nu},
$$


где резольвента $R_{\nu}(T)$ представима в виде сходящегося всюду в $\mathbb{H}$ ряда Неймана

$$
\begin{gathered}
R_{\nu}(T):=-\frac{1}{q(x)+\nu}\left(\mathbb{I}+\frac{q^{\prime \prime}(x)}{q(x)+\nu} M_{0}^{-1}+\left(\frac{q^{\prime \prime}(x)}{q(x)+\nu} M_{0}^{-1}\right)^{2}+\ldots+\right. \\
\left.\left(\frac{q^{\prime \prime}(x)}{q(x)+\nu} M_{0}^{-1}\right)^{n}+\ldots\right),
\end{gathered}
$$

если $\nu>0$ взять настолько большим, чтобы

$$
\left\|\frac{q^{\prime \prime}(x)}{q(x)+\nu} M_{0}^{-1}\right\|<1
$$

В результате для любого $\phi(x) \in \mathbb{H}$ имеем такое представление:

$$
R_{\nu}(T) \phi(x)=\Psi_{0}(x) \phi(x)+\Psi_{1}(x)
$$

где

$$
\begin{gathered}
\Psi_{0}(x)=-\frac{1}{q(x)+\nu} \\
\Psi_{1}(x)=-\frac{1}{q(x)+\nu}\left(\frac{q^{\prime \prime}(x)}{q(x)+\nu} M_{0}^{-1}+\left(\frac{q^{\prime \prime}(x)}{q(x)+\nu} M_{0}^{-1}\right)^{2}+\ldots\right. \\
\left.+\left(\frac{q^{\prime \prime}(x)}{q(x)+\nu} M_{0}^{-1}\right)^{n}+\ldots\right) \phi(x)
\end{gathered}
$$

и $\Psi_{0}(x) \in \mathbb{C}^{2}[-1,1], \Psi_{1}(x) \in \mathbb{C}[-1,1], \Psi_{1}( \pm 1)=0$. А с учётом уравнения $R_{\nu}(T)=\tilde{c} \mathbb{I}$ получаем

$$
\Psi_{1}(x)-\frac{1}{q(x)+\nu} \phi(x)=\frac{1}{c-\nu} \phi(x), \quad \phi(x) \in \mathbb{H},
$$

откуда и из (4) легко вывести равенство

$$
(\nu-c) \sum_{i=1}^{\infty}\left(\frac{q^{\prime \prime}(x)}{q(x)+\nu} M_{0}^{-1}\right)^{i} \phi(x)=q^{\prime \prime}(x) M_{0}^{-1} \phi(x),
$$

которое совместно с (4) выполняется лишь для $\phi(x) \equiv 0$ (или п.в. $\phi(x) \equiv$ $0)$ на $[-1,1]$, так как слева стоит выражение, зависящее от произвольного достаточно большого $\nu$, а справа этого параметра нет. 
Таким образом, спектр Е задачи Рэлея (1)-(2) не содержит ни одного собственного числа, то есть дискретной его части.

Пусть $a_{0}=\min _{x \in[-1,1]} q(x), b_{0}=\max _{x \in[-1,1]} q(x)$. Так как резольвента $R_{c}(T)$ существует для любого комплексного $c$, то остается показать, что отрезок $\left[a_{0}, b_{0}\right]$ заполняют только точки остаточной части спектра оператора $T$, а вне этого отрезка расположены лишь регулярные точки.

Итак, (п.в.) в достаточно малой ненулевой окрестности любой точки $x_{0} \in[-1,1]$ произвольная функция $y(x) \in \mathbb{H}$ всвязи с интегральной нормой пространства $\mathbb{H}$ ведет себя примерно как $C\left(y(x), x_{0}\right)\left(x-x_{0}\right)^{\gamma\left(y(x), x_{0}\right)}$ $\left(C\left(y(x), x_{0}\right), \gamma\left(y(x), x_{0}\right)\right.$ - действительные числа, зависящие от функции $y(x)$ и точки $\left.x_{0}, \gamma\left(y(x), x_{0}\right)>-1 / 2\right)$. А (п.в.) в достаточно малой ненулевой окрестности нуля $\tilde{x}_{0} \in[-1,1]$ функции $\varphi(x)=q(x)-c$ $\left(c \in\left[a_{0}, b_{0}\right]\right)$ всвязи с представлением $(6) \tilde{y}(x) \in \mathbb{H}$ ведет себя примерно как $C\left(\tilde{y}(x), \tilde{x_{0}}\right)\left(x-\tilde{x_{0}}\right)^{\gamma\left(\tilde{y}(x), \tilde{x_{0}}\right)}(q(x)-c)\left(C\left(\tilde{y}(x), \tilde{x_{0}}\right), \gamma\left(\tilde{y}(x), \tilde{x_{0}}\right)\right.$ - действительные числа, зависящие от функции $\tilde{y}(x)$ и точки $\tilde{x}_{0}, \gamma\left(\tilde{y}(x), \tilde{x}_{0}\right)>$ $-1 / 2)$. То есть, для любого числа $c \in\left[a_{0}, b_{0}\right]$ область определения резольвенты $R_{c}(T)$ не является всюду плотной в $\mathbb{H}$ (см. [2, с. 260-262]). Другими словами, любое число $c \in\left[a_{0}, b_{0}\right]$ принадлежит остаточной части спектра оператора $T$. Из представления (6) и свойства А следует, что любое комплексное число $c \notin\left[a_{0}, b_{0}\right]$ является регулярной точкой оператора $T$.

В результате доказано следующее утверждение.

Утверждение 1 Пусть $q(x)$ - многочлен ненулевой степени такой, что выполняется условие (3), и \pm - спектр задачи Рэлея (1)-(2). Тогда Е составляют все точки отрезка с концами, равными числам $-\alpha a_{0}$ $u-\alpha b_{0}$. Причем этот отрезок заполняют лишь точки остаточной части спектра.

Здесъ $a_{0}=\min _{x \in[-1,1]} q(x), b_{0}=\max _{x \in[-1,1]} q(x), \alpha-$ волновое число.

\section{3. ЗАКЛЮЧЕНИЕ}

Метод, изложенный в работе, можно коротко охарактеризовать как 
резольвентный. Спектр задачи Рэлея при условии, что профиль скорости - многочлен, не содержит собственных чисел, а содержит лишь остаточную часть спектра. Этого и следовало ожидать, так как рассматривать устойчивость или неустойчивость движения абсолютно кинематически не вязкой жидкости $(R=\infty)$ не приходится.

\section{Список литературы}

[1] Наймарк M. А. Линейные дифференциальные операторы / М. А. Наймарк. - М. : Гос. издат. технико-теоретической лит., 1954. - 352 c.

[2] Садовничий В. А. Теория операторов / В. А. Садовничий. - изд. 2 М. : Изд-во Моск. ун-та, 1986. - 386 с. 